\title{
Accuracy and finish during wire electric discharge machining (EDM) of metal matrix composites (MMCs) for different reinforcement size and machining conditions
}

\author{
A. Pramanik ${ }^{1 *}$, M.N. Islam ${ }^{1}$, B. Boswell ${ }^{1}$, A. K. Basak ${ }^{2}$, Yu Dong ${ }^{1}$, G. Littlefair ${ }^{3}$ \\ ${ }^{1}$ Department of Mechanical Engineering, Curtin University, Bentley, WA, Australia \\ ${ }^{2}$ Adelaide microscopy, the University of Adelaide, Adelaide, SA, Australia \\ ${ }^{3}$ School of Engineering, Deakin University, Waurn Ponds, Australia
}

*Corresponding author, Email: akprama@yahoo.com, Phone: +61 89266 7981, Fax: +61 892662681.

\begin{abstract}
Electric discharge machining (EDM) has been established as an effective alternative process to conventional material removal processes for machining reinforced metal matrix composites (MMCs). Wire cut EDM holes were produced in a MMCs (10 vol\% of $\mathrm{SiC}$ in $6061 \mathrm{Al})$, which were then investigated to determine the machinability of the material using this process. It was observed that the input factors such as the size of reinforced particles, wire tension, and pulse on time, significantly affect diameter error, circularity and surface roughness. Pulse on time, the interaction between pulse on time and wire tension contribute to the maximum diameter error. The wire tension is the most significant factor to circularity, which is followed by the interaction between pulse on time. In particular, wire tension with low and high tensions results in poor circularity. It has been found that there are more surface defects encountered when particle sizes are smaller, and circularity is improved when particles are in a medium size. In addition, the surface defect is reduced as the particles increase the melting resistance of the surface. The higher pulse on time leads to higher heat and more time to degrade the surface. Therefore, low pulse on time and wire tension gave better surface finish.
\end{abstract}

Key words: Metal matric composites (MMCs), electric discharge machining (EDM), particle size, pulse on time, wire tension, dimensional error and surface roughness.

\section{Introduction}

Metal alloys that are increasingly hard to be machined are currently being used to produce components, as these material possess desirable qualities required by aerospace and automobile industries. Non- 
traditional manufacturing processes are gradually used to produce these parts, particularly via wire EDM. Components to be cut are submersed in a dielectric liquid, where an electrical discharge takes place [1]. A discharge channel is created when the temperature reaches approximately $12,000{ }^{\circ} \mathrm{C}$, removing material by evaporation and melting, which is flushed away by the dielectric fluid. A heat affective zone (HAZ) is formed when the discharge ceases, due to the high cooling rate on the workpiece surface. EDM is governed by Faradays Law, and the resulting thermal effect not only removes material from the workpiece but also changes metallurgical constituents at the HAZ [2, 3].

The high cost of machining MMCs has reduced the application uptake of these advanced materials [4, 5]. Typically to date they have been machined with either an electroplated diamond-grinding wheel or carbide / poly crystalline diamond cutting tools for a conventional machining process. Non-contact material removal processes such as wire EDM is an attractive alternative [6] due to its eliminating the requirement for expensive tooling [7, 8]. research on the EDM process for MMCs components includes the assessment of die sink EDM MMCs reinforced with $\mathrm{Al}_{2} \mathrm{O}_{3}$ as well as corresponding matrix material (A16061), as mentioned Mouangue et al., [9]. It is suggested that the tool wear ration and material removal rate are acceptable at low peak current, short time and longer off time. The performance of wire EDM cutting MMCs is less effective when compared with Al6061 material because the removal rate is less and thus the tool wear is increased. Also the off time increases as opposed to Al6061 material. Singh et al. [10] implemented the analysis of variance (ANOVA) and established the optimal levels for maximizing the responses. It was noted that material removal rate was found to be higher for larger current and pulse ON-time settings at the expense of side taper, radial overcut and surface finish. Tool wear rate for MMCs was also found to increase for larger current settings. Garg et al. [6] reviewed sinking EDM, WEDM, and powder-mixed EDM of MMCs, all of which were intended to enhance the capability of machining performance, and to improve output and working conditions. Khan and Singh [11] performed EDM on MMCs (i.e. Al/SiC composites) by non-rotating and rotating electrode. Material removal rate and electrode wear rate were the response variables in the cutting tests, and input parameters were pulse on time, pulse off time, voltage and peak current. Results show that material removal rate and tool wear rate are increased for rotating electrodes. Lal et al., [12] investigated the effect of pulse on time, pulse off time, pulse current and wire drum speed on the material removal rate for $\mathrm{A} 17075 / 7.5 \% \mathrm{SiC} / 7.5 \% \mathrm{Al}_{2} \mathrm{O}_{3} \mathrm{MMCs}$. Taguchi method was used for parameter optimization and the level of importance was determined from ANOVA. The pulse on time was the most significant parameter that contributed to the maximum $(46.04 \%)$ the material removal rate $(46.04 \%)$, followed by pulse current $(34.72 \%)$, pulse off time $(10.23 \%)$ and interaction between pulse on time and pulse off time $(5.46 \%)$. 
The wire drum speed had insignificant effect on the material removal rate. Tian et al., [13] introduced a probabilistic design system to analyze the effects of machining parameters during EDM. Monte Carlo simulation method and response surface method were both used in the sensitivity analysis. Discharge voltage, peak current, pulse-on time and discharge channel radius are selected as design variables. The results of sensitivity analysis meet the confidence limit of 0.95 . The discharge voltage and peak current significantly affect the EDM process, whereas the pulse-on time and discharge channel radius have minor influence. Moreover, the increase in discharge channel radius can reduce the material removal rate. Conversely, the increase in other parameters can increase the material removal rate. Amini et al., [14] used an L32 orthogonal array based on the Taguchi method for design of experiments. Process modelling was carried out by using a multilayer neural network to determine the most effective parameters on material removal rate and surface roughness. On the other hand, a genetic algorithm was used to optimize the process performance of WEDM. The optimization results are shown to be in good agreement with experimental outputs. Rozenek et al., [15] showed the effects of discharge current, pulse-on time, pulseoff time, voltage on the machining feed rate during wire EDM of MMCs reinforced with $\mathrm{SiC}$ and $\mathrm{Al}_{2} \mathrm{O}_{3}$ particles in AlSi7Mg alloy. The characteristics of wire EDM of MMCs are similar to those of the base material (AlSi7Mg aluminum alloy). The maximum cutting speed for $\mathrm{AlSi7Mg} / \mathrm{SiC}$ and $\mathrm{AlSi} 7 \mathrm{Mg} / \mathrm{Al}_{2} \mathrm{O}_{3}$ composites materials are approximately 3 times and 6.5 times lower than the cutting speed of aluminum alloys, respectively.

Little research in analyzing diameter error and circularity during wire EDM of MMCs reinforced with particles of different sizes has been discussed in previous literature review. Diameter error and circularity are regarded as important parameters in defining dimensional accuracy and tolerance of machined components. As a consequence, this research is to investigate the effects of reinforcement size, pulse on time, and wire tension on the diameter error, circularity and surface roughness during wire EDM machining of MMCs.

\section{Scope}

The accuracy specification of machined holes is normally represented by their diameter error and circularity as the most important parameters. Diameter error is the difference between the measured diameter and the designed diameter, where a positive error indicates overcutting of the hole. When cylindrical fit is necessary, diameter error is the most important characteristic of a machined hole. Circularity, also known as roundness, is another important quality characteristic. Circularity is defined by two concentric circular boundaries, within which each circular element of the surface must lie [16]. It 
is particularly important for rotating component parts where excessive circularity values may cause unacceptable vibration and heat. Another important quality parameter for examining is surface finish, as it is significant with respect to wear, corrosion, fatigue, noise, load-carrying capacity, heat transfer, and many others. Surface roughness represents the random and repetitive deviations of a surface profile from the nominal surface, and can be expressed by a number of factors. Unfortunately, no single factor appears to be capable of adequately describing the surface quality. For convenience, the arithmetic average was adopted to represent surface roughness, since it is commonly used and is internationally accepted. Therefore, these quality parameters were employed to study the suitability of using wire EDM machine to produce holes in MMC workpieces.

The quality parameter data were analysed by applying two statics techniques including Pareto ANOVA and Taguchi's signal-to-noise (S/N) ratio analysis. In the traditional ANOVA analysis, average response values have been used. This strategy is particularly suitable for monitoring trends or changes in the relationship of variables. However, it does not provide the complete representation because it normally does not include data on the response scattering. The Pareto ANOVA is a method for determining the contribution of each input parameter, and their interactions with output quality parameters. Further details on Pareto ANOVA can be found in Park [17].

To optimise the robustness of manufacturing process data, the Taguchi statistical method is used in applying signal-to-noise $(S / N)$ ratio to the data. The $\mathrm{S} / \mathrm{N}$ ratio can be calculated using the following formula based on 'the smaller the better' criterion [18]:

$$
S / N=-10 \log \frac{1}{n}\left(\sum_{i=1}^{n} y_{i}^{2}\right)
$$

where $n$ is the number of observations and $y$ is the observed data.

The higher the value of the $\mathrm{S} / \mathrm{N}$ ratio, the better the result becomes since it guarantees the highest quality with a minimum variance. An expanded explanation of the Taguchi method can be found elsewhere in [18]. This research is limited to MMCs with three different reinforcement sizes - $0.7,3$ and $13 \mu \mathrm{m}$ as there is a wide range of materials available for turning operations. 


\section{Experiment Procedure}

The hole cutting test data were obtained from a FANUC ROBOCUT $\alpha-0 \mathrm{iD}$ wire EDM machine with a fixed input condition for each test. The machining conditions were:

- wire speed: $10 \mathrm{~m} / \mathrm{min}$

- flushing rate: $10 \mathrm{~L} / \mathrm{min}$

- open circuit voltage: $85 \mathrm{~V}$

- servo voltage: $44 \mathrm{~V}$

- wire electrode Zinc coated brass wire of diameter: $0.25 \mathrm{~mm}$

- reinforced particle size: $0.7,3$ and $13 \mu \mathrm{m}$

- wire tension: 600,1200 and $1800 \mathrm{gf}$

- pulse on time: 2,3 and $4 \mu \mathrm{s}$

The experimental test procedure was prearranged by using Taguchi's $\mathrm{L}_{27}\left(3^{13}\right)$ orthogonal array, ensuring all the parameters were robustly examined. A copy of $\mathrm{L}_{27}\left(3^{13}\right)$ array is available in Taguchi [19]. A total of 27 holes were machined, namely nine holes for each of three particle reinforced MMCs. The output quality parameters were measured for diameter error, circularity and surface roughness.

The workpieces in cutting tests were made from a rectangular plate of MMCs with the dimensions shown in Figure 1. Each workpiece is used for nine tests of different combinations of control parameters for each test. The control parameters and their levels used for analysis of first three groups are shown in table 1 , and the details of all experimental tests are given in table 2.

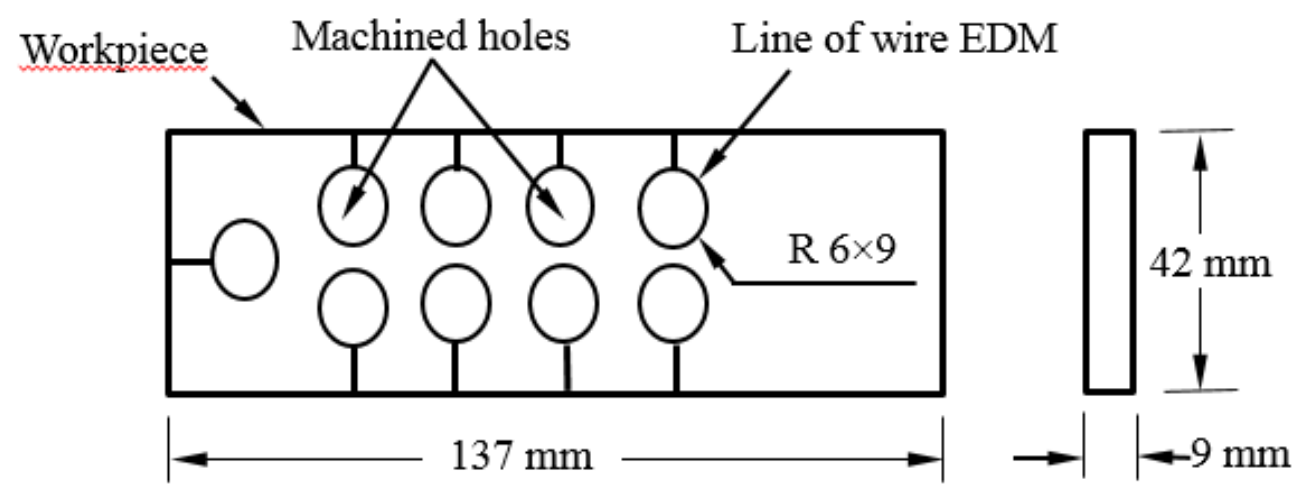

Fig. 1 Dimensions of a workpiece and holes 
Table 1 Input parameters with their Levels

\begin{tabular}{ccccccc}
\hline Input parameter & & Symbol & & Level 0 & Level 1 & Level 2 \\
\cline { 1 - 3 } \cline { 5 - 7 } Particle size $(\mu \mathrm{m})$ & & $\mathrm{A}$ & & 0.7 & 3 & 13 \\
Wire Tension $(\mathrm{gf})$ & & $\mathrm{B}$ & & 600 & 1200 & 1800 \\
Pulse on Time $(\mu \mathrm{s})$ & & $\mathrm{C}$ & & 2 & 3 & 4 \\
\hline
\end{tabular}

Table 2 Experiments details

\begin{tabular}{cccc} 
Expt. no. & Particle size $(\boldsymbol{\mu m})$ & Wire Tension $(\mathbf{g f})$ & Pulse on Time $(\boldsymbol{\mu s})$ \\
\hline 1 & 0.7 & 600 & 2 \\
2 & 0.7 & 600 & 3 \\
3 & 0.7 & 600 & 4 \\
4 & 0.7 & 1200 & 2 \\
5 & 0.7 & 1200 & 3 \\
6 & 0.7 & 1200 & 4 \\
7 & 0.7 & 1800 & 2 \\
8 & 0.7 & 1800 & 3 \\
9 & 0.7 & 1800 & 4 \\
10 & 3 & 600 & 2 \\
11 & 3 & 600 & 3 \\
12 & 3 & 600 & 4 \\
13 & 3 & 1200 & 2 \\
14 & 3 & 1200 & 3 \\
15 & 3 & 1200 & 4 \\
16 & 3 & 1800 & 2 \\
17 & 3 & 1800 & 3 \\
18 & 3 & 1800 & 4 \\
19 & 13 & 600 & 2 \\
20 & 13 & 600 & 3 \\
21 & 13 & 600 & 4 \\
22 & 13 & 1200 & 2 \\
23 & 13 & 1200 & 3 \\
24 & 13 & 1200 & 4 \\
25 & 13 & 1800 & 2 \\
26 & 13 & 1800 & 4 \\
27 & 13 & 1800 &
\end{tabular}

A Discovery Model D-8 coordinate measuring machine (CMM), was used to determine the precision of the cut hole, using the Renishaw probe in a star configuration for convenience. The diameters of cut holes were determined by using the standard built-in software of the CMM. Eight coordinate points were measured for establishing the diameter, and each coordinate measurement was repeated three times. This 
coordinate data was also used to find the circularity of every cut hole. The surface roughness parameter for each turned surface was determined (arithmetic average $\left(R_{a}\right)$ ) by using a tally surf Surftest SJ-201P.

\section{Results \& analysis}

Due to the substantial amount of coordinate data and space constraints, only a sample of coordinate data has been presented in this paper. For the analysis of the workpieces, all the data were considered. The multi-variability of data obtained allowed workpiece data to be present in a number of ways, with a Pareto ANOVA being conducted on all quality test parameters. The adopted format was chosen carefully to maximise the clarity of the presentation. Experimental results for diameter error, circularity, surface roughness and their corresponding $\mathrm{S} / \mathrm{N}$ ratios are summarised in Table 3.

\subsection{Diameter Error}

The Pareto AVOVA analysis for diameter error given in Table 4 reveals that pulse on time (C) has the most significant effect on diameter error with a influence ratio $(\mathrm{P} \cong 25.25 \%)$, followed by the interaction between wire tension and pulse on time $(B \times C)(P \cong 25.19 \%)$ which has almost similar contribution to that of pulse on time. The contributions of particle size (A) and, the interaction between particle size and wire tension $(\mathrm{A} \times \mathrm{B})$ are almost comparable (9.87 and $9.04 \%$ respectively). It is worth noting that the total influence of main effects is about $41.40 \%$, compared to total impact of the interaction effects at $59.60 \%$, thus making it difficult to optimize the diameter error by the selection of input parameters.

Table 1 Results for diameter error, circularity, surface roughness and corresponding S/N Ratios

\begin{tabular}{|c|c|c|c|c|c|c|}
\hline \multirow[b]{2}{*}{$\begin{array}{l}\text { Expt } \\
\text { no }\end{array}$} & \multicolumn{3}{|c|}{ Measured parameters } & \multicolumn{3}{|c|}{ Calculated $\mathrm{S} / \mathrm{N}$ ratio for } \\
\hline & $\begin{array}{l}\text { Diameter } \\
\text { error } \\
(\mathrm{mm})\end{array}$ & $\begin{array}{l}\text { Circularity } \\
(\mathrm{mm})\end{array}$ & $\begin{array}{l}\text { Roughness } \\
(\mu \mathrm{m})\end{array}$ & $\begin{array}{l}\text { Diameter } \\
\text { error }(\mathrm{dB})\end{array}$ & $\begin{array}{l}\text { Circularity } \\
(\mathrm{dB})\end{array}$ & $\begin{array}{l}\text { Roughness } \\
\text { (dB) }\end{array}$ \\
\hline 1 & 0.075 & 0.006 & 2.124 & 22.536 & 44.815 & -6.550 \\
\hline 2 & 0.086 & 0.008 & 2.671 & 21.343 & 41.761 & -8.538 \\
\hline 3 & 0.092 & 0.008 & 2.628 & 20.754 & 42.194 & -8.397 \\
\hline 4 & 0.081 & 0.009 & 2.294 & 21.793 & 41.192 & -7.217 \\
\hline 5 & 0.090 & 0.007 & 2.418 & 20.946 & 43.039 & -7.674 \\
\hline 6 & 0.096 & 0.007 & 2.720 & 20.354 & 43.436 & -8.695 \\
\hline 7 & 0.083 & 0.006 & 2.372 & 21.582 & 43.943 & -7.507 \\
\hline 8 & 0.089 & 0.007 & 2.754 & 21.010 & 43.098 & -8.803 \\
\hline 9 & 0.093 & 0.008 & 2.521 & 20.598 & 41.871 & -8.036 \\
\hline 10 & 0.085 & 0.006 & 2.094 & 21.441 & 44.815 & -6.426 \\
\hline 11 & 0.094 & 0.006 & 2.223 & 20.565 & 44.397 & -6.945 \\
\hline
\end{tabular}




\begin{tabular}{lllllll}
12 & 0.114 & 0.011 & 2.679 & 18.180 & 39.148 & -8.563 \\
13 & 0.096 & 0.008 & 2.159 & 20.383 & 42.007 & -6.691 \\
14 & 0.102 & 0.066 & 3.097 & 19.856 & 23.597 & -9.822 \\
15 & 0.063 & 0.012 & 2.737 & 23.963 & 38.396 & -8.749 \\
16 & 0.095 & 0.008 & 2.152 & 20.412 & 41.893 & -6.663 \\
17 & 0.099 & 0.007 & 2.368 & 20.115 & 42.570 & -7.493 \\
18 & 0.105 & 0.008 & 2.094 & 19.574 & 42.243 & -6.426 \\
19 & 0.036 & 0.006 & 1.921 & 23.060 & 44.357 & -5.678 \\
20 & 0.095 & 0.009 & 2.181 & 20.439 & 41.192 & -6.779 \\
21 & 0.101 & 0.008 & 2.172 & 19.910 & 41.407 & -6.743 \\
22 & 0.085 & 0.012 & 2.159 & 21.373 & 38.633 & -6.691 \\
23 & 0.090 & 0.016 & 2.476 & 20.915 & 35.912 & -7.880 \\
24 & 0.094 & 0.016 & 2.066 & 20.506 & 33.556 & -6.309 \\
25 & 0.090 & 0.011 & 2.062 & 20.934 & 37.764 & -6.293 \\
26 & 0.095 & 0.007 & 2.076 & 20.413 & 43.010 & -6.351 \\
27 & 0.100 & 0.007 & 2.320 & 20.028 & 43.500 & -7.315 \\
\hline
\end{tabular}

The response table and graphs for diameter error are shown in Table 5 and Figure 2, respectively, with the slopes of response graphs, representing the strength of the influence as confirmed by the ANOVA analysis in Table 4. The low particle size (A0) was the best particle size to achieve a low diameter error as shown in Table 5. Since the interaction of $\mathrm{B} \times \mathrm{C}$ is significant, the $\mathrm{B} \times \mathrm{C}$ two-way table has been applied to select the optimum levels of B and C (see Appendix). The optimum combination of factors B and C in order to achieve the lowest diameter error was determined as B0C0. Therefore the best combination of input variables for minimising diameter was determined as A0B0C0, which is based on a low level of reinforcement size $(0.3 \mu \mathrm{m})$, lowest level of wire tension (600 gf), and low level of pulse on time $(2 \mu \mathrm{s})$. 
Table 4 Pareto ANOVA analysis for diameter error

\begin{tabular}{|c|c|c|c|c|c|c|c|c|c|}
\hline \multirow[t]{2}{*}{ Sum at factor level } & \multicolumn{9}{|c|}{ Factor and interaction } \\
\hline & A & $\mathrm{B}$ & $\mathrm{A} \times \mathrm{B}$ & $\mathrm{A} \times \mathrm{B}$ & $\mathrm{C}$ & $\mathrm{A} \times \mathrm{C}$ & $\mathrm{A} \times \mathrm{C}$ & $\mathrm{B} \times \mathrm{C}$ & $\mathrm{B} \times \mathrm{C}$ \\
\hline 0 & 190.92 & 188.23 & 187.5 & 190.2 & 193.5 & 189.3 & 186.8 & 193.4 & 188.9 \\
\hline 1 & 184.49 & 190.09 & 184.6 & 186.6 & 185.6 & 185.9 & 190.3 & 186.1 & 190.1 \\
\hline 2 & 187.58 & 184.67 & 190.8 & 186.1 & 183.8 & 187.6 & 185.7 & 183.4 & 183.9 \\
\hline $\begin{array}{l}\text { Sum of squares of } \\
\text { difference }(S)\end{array}$ & 62.00 & 45.55 & 56.78 & 29.54 & 158.6 & 17.51 & 35.41 & 158.3 & 64.59 \\
\hline $\begin{array}{l}\text { Contribution ratio } \\
(\%)\end{array}$ & 9.87 & 7.25 & 9.04 & 4.70 & 25.25 & 2.79 & 5.63 & 25.19 & 10.28 \\
\hline $\begin{array}{l}\text { Cumulative } \\
\text { contribution }\end{array}$ & 25.25 & 50.45 & 60.72 & 70.59 & 79.63 & 86.88 & 92.51 & 97.21 & 100.0 \\
\hline $\begin{array}{l}\text { Check on significant } \\
\text { interaction }\end{array}$ & \multicolumn{9}{|c|}{ B x C two-way table } \\
\hline $\begin{array}{l}\text { Optimum } \\
\text { combination of } \\
\text { significant factor } \\
\text { level }\end{array}$ & \multicolumn{9}{|c|}{$\mathrm{A} 0 \mathrm{~B} 0 \mathrm{C} 0$} \\
\hline
\end{tabular}

Table 5 Response table for mean $\mathrm{S} / \mathrm{N}$ ratio for diameter error and significant interaction

\begin{tabular}{llllll}
\hline Input parameter & Symbol & Level 0 & Level 1 & Level 2 & max-min \\
\hline Particle size & $\mathrm{A}$ & 21.21 & 20.50 & 20.84 & 0.71 \\
Wire Tension & $\mathrm{B}$ & 20.91 & 21.12 & 20.52 & 0.60 \\
Pulse on Time & $\mathrm{C}$ & 21.50 & 20.62 & 20.43 & 1.07 \\
Interaction & $\mathrm{BXC}$ & 21.49 & 20.68 & 20.39 & 1.10 \\
\hline
\end{tabular}




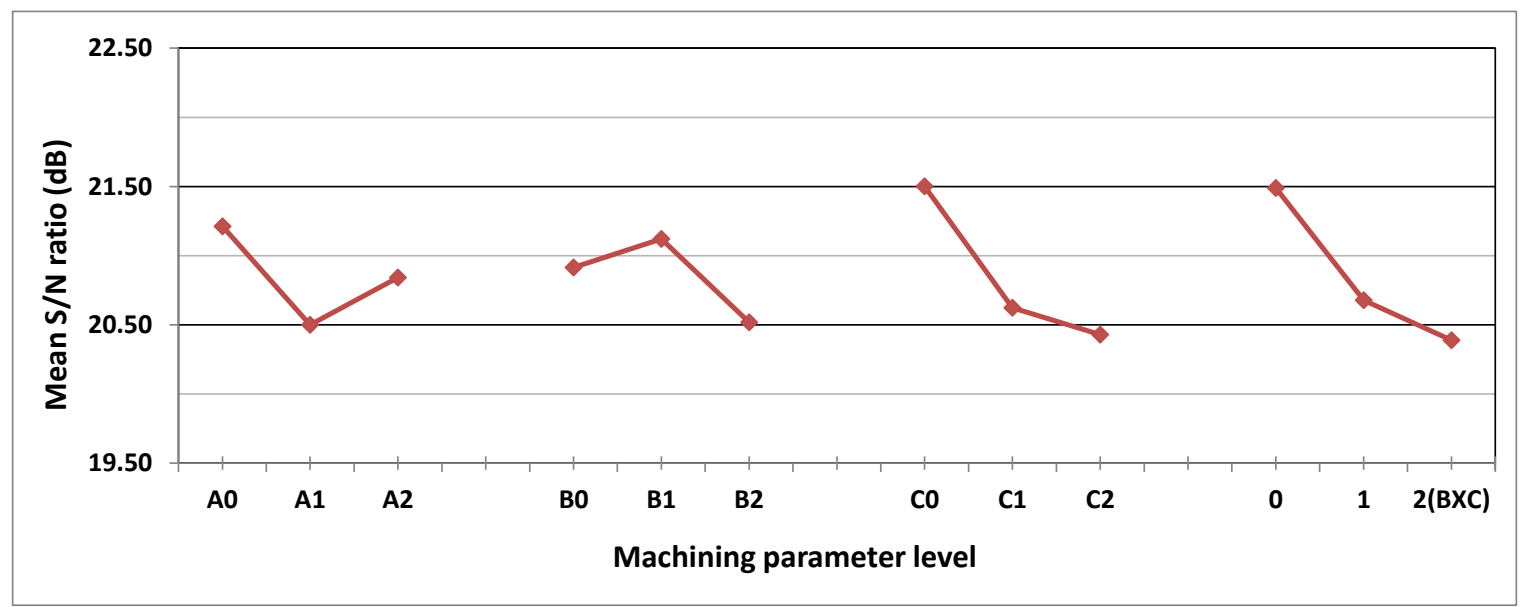

Fig. 2 Response graph of $\mathrm{S} / \mathrm{N}$ ratios for Diameter Error

The average variation of diameter error as the traditional method for analysing the diameter error illustrates that the smaller pulse on time gives smaller errors as shown in Figure 3. Similarly, the error decreases with the decrease of wire tension, and it is the lowest at lowest wire tension (600 gf). As illustrated in Figure 3, a similar conclusion can be drawn through the Taguchi method (A0B0C0). It is evidently seen that the diameter error has a minimum range.

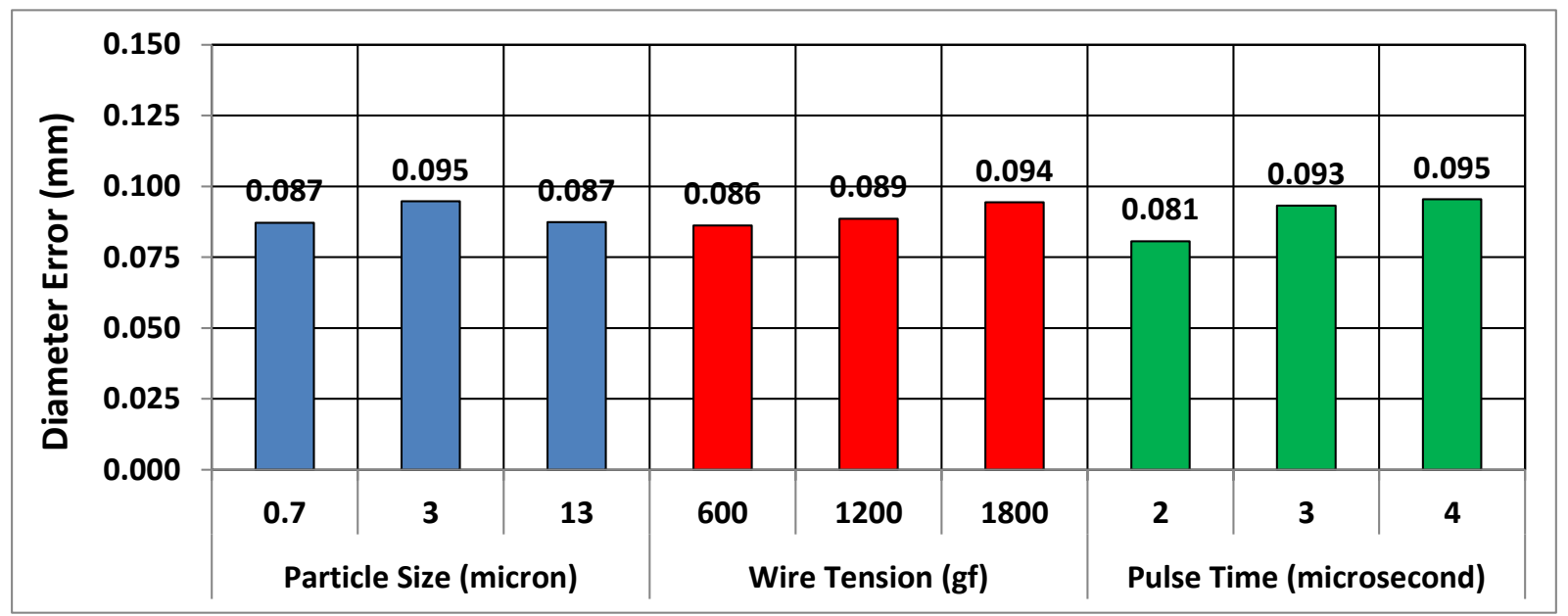

Fig. 3 Average diameter errors for three parameters

\subsection{Circularity}

The Pareto ANOVA analysis for circularity given in Table 6 indicates that wire tension (B) has the most significant effect on circularity with a contribution ratio $(\mathrm{P} \cong 36 \%)$, followed by the interaction between pulse on time and wire tension $(\mathrm{B} \times \mathrm{C})(\mathrm{P} \cong 17 \%)$, particle size $(\mathrm{A})(\mathrm{P} \cong 14 \%)$ and, interaction between 
particle size and wire tension $(\mathrm{A} \times \mathrm{B})(\mathrm{P} \cong 9 \%$ and $7 \%)$. The connections between reinforcement size and wire tension $(\mathrm{A} \times \mathrm{B})$, and between reinforcement size and pulse on time $(\mathrm{A} \times \mathrm{C})$ also played a part, with an influence of $10.81 \%$ and $10.45 \%$, respectively. The total contribution of main effects is about $50 \%$, compared to that of the interaction effects of $50 \%$, also making it difficult to optimise the circularity error by the selection of input parameters.

Response graph in Figure 4 confirms the findings of the Pareto ANOVA given in Table 6. The low level of reinforcement size (A0) is the best combination as shown in Table 7. As the interaction $\mathrm{B} \times \mathrm{C}$ was significant, and $\mathrm{B} \times \mathrm{C}$ two-way table was applied to select their levels (see Appendix). From the $\mathrm{B} \times \mathrm{C}$ twoway table, the optimum combination of factors $\mathrm{B}$ and $\mathrm{C}$ in order to achieve the best circularity was determined as $\mathrm{B} 0 \mathrm{C} 0$. Consequently, the best combination of input variables for minimising circularity was determined as $\mathrm{A} 0 \mathrm{~B} 0 \mathrm{C} 0$; having a low level of reinforcement size $(0.3 \mu \mathrm{m})$, the lowest level of wire tension (600 gf), and a low level of pulse on time $(2 \mu \mathrm{s})$.

Table 6 Pareto ANOVA for circularity

\begin{tabular}{|c|c|c|c|c|c|c|c|c|c|}
\hline \multirow[t]{2}{*}{ Sum at factor level } & \multicolumn{9}{|c|}{ Factor and interaction } \\
\hline & $\mathrm{A}$ & $\mathrm{B}$ & $\mathrm{A} \times \mathrm{B}$ & $\mathrm{A} \times \mathrm{B}$ & $\mathrm{C}$ & $\mathrm{A} \times \mathrm{C}$ & $\mathrm{A} \times \mathrm{C}$ & $\mathrm{B} \times \mathrm{C}$ & $\mathrm{B} \times \mathrm{C}$ \\
\hline 0 & 385.3 & 384.0 & 363.5 & 357.0 & 379.4 & 369.8 & 358.9 & 378.0 & 364.1 \\
\hline 1 & 359.0 & 339.7 & 380.3 & 381.3 & 358.5 & 375.0 & 368.4 & 376.8 & 366.3 \\
\hline 2 & 359.3 & 379.8 & 359.8 & 365.3 & 365.7 & 358.8 & 376.3 & 348.9 & 373.2 \\
\hline $\begin{array}{l}\text { Sum of squares of } \\
\text { difference }(S)\end{array}$ & 1367.8 & 3591.6 & 710.9 & 913.7 & 672.7 & 413.3 & 452.9 & 1630 & 135.6 \\
\hline $\begin{array}{l}\text { Contribution ratio } \\
(\%)\end{array}$ & 13.83 & 36.32 & 7.19 & 9.24 & 6.80 & 4.18 & 4.58 & 16.48 & 1.37 \\
\hline $\begin{array}{l}\text { Cumulative } \\
\text { contribution }\end{array}$ & 36.32 & 52.80 & 66.64 & 75.88 & 83.07 & 89.87 & 94.45 & 98.63 & 100.0 \\
\hline $\begin{array}{l}\text { Check on significant } \\
\text { interaction }\end{array}$ & \multicolumn{9}{|c|}{ B x C two-way table } \\
\hline $\begin{array}{l}\text { Optimum } \\
\text { combination of } \\
\text { significant factor } \\
\text { level }\end{array}$ & \multicolumn{9}{|c|}{$\mathrm{A} 0 \mathrm{~B} 0 \mathrm{C} 0$} \\
\hline
\end{tabular}


Table 7 Response table for mean S/N ratio for circularity error and significant interaction

\begin{tabular}{llllll}
\hline Input parameter & Symbol & Level 0 & Level 1 & Level 2 & max-min \\
\hline Particle size & $\mathrm{A}$ & 42.82 & 39.90 & 39.93 & 2.92 \\
Wire Tension & $\mathrm{B}$ & 42.68 & 37.75 & 42.21 & 4.92 \\
Pulse on Time & $\mathrm{C}$ & 42.16 & 39.84 & 40.64 & 2.32 \\
Interaction & $\mathrm{BXC}$ & 42.01 & 41.87 & 38.77 & 3.24 \\
\hline
\end{tabular}

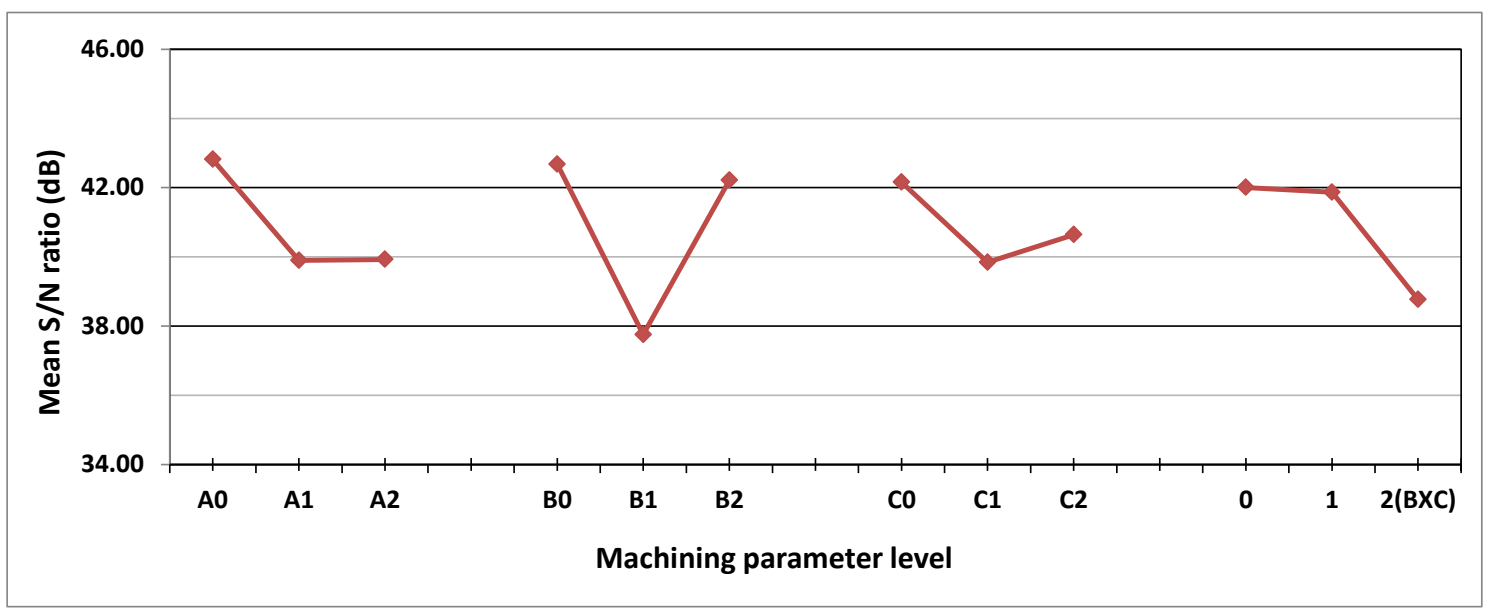

Fig. 4 Response graph of $\mathrm{S} / \mathrm{N}$ ratios for circularity

The tradition analyses for circularity are presented in Figure 5, which shows the average variation of circularity. The low level of particle size $(0.7 \mu \mathrm{m})$, low level of wire tension $(600 \mathrm{gf})$ and low level of pulse on time $(2 \mu \mathrm{s})$ gives the best result. The analysis of Figure 5 leads to the similar conclusion reached through the Taguchi method.

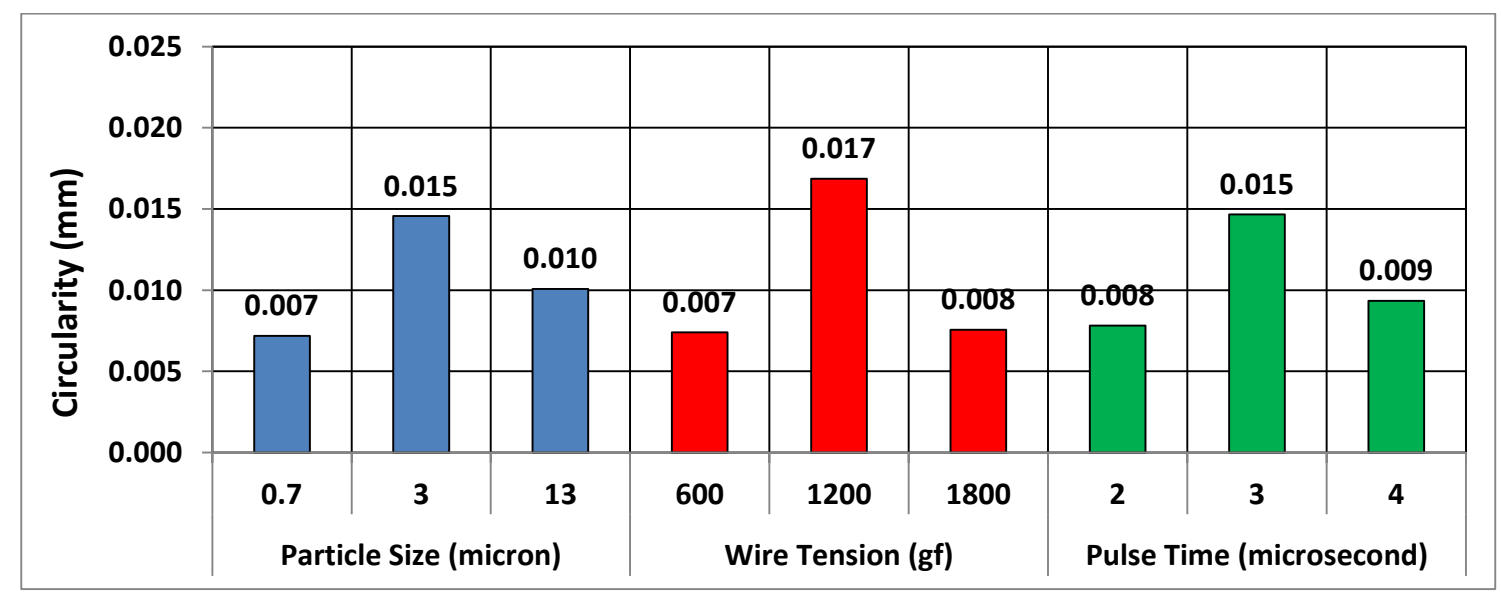

Fig. 5 Average circularity for three parameters 


\subsection{Surface Roughness}

The Pareto AVOVA for surface roughness given in Table 8 signifies that pulse on time (C) and reinforcement size (A) have the most significant effect on surface roughness. Having almost equal contribution ratio $(\mathrm{P} \cong 34 \%)$, followed by wire tension $(\mathrm{B})(\mathrm{P} \cong 8 \%)$, the interaction between reinforcement size and wire tension $(\mathrm{A} \times \mathrm{B})(\mathrm{P} \cong 8 \%)$ as well as the interaction between wire tension and pulse on time $(\mathrm{B} \times \mathrm{C})(\mathrm{P} \cong 8 \%)$. The total impact of main effects is about $77.07 \%$ compared to the total contribution of interaction effects of $22.93 \%$. Therefore, it becomes relatively easy to optimise the surface roughness in this instance by the selection of input parameters.

The response graph in Figure 6 confirms the findings of the Pareto ANOVA given in Table 8. Table 9 shows that the low level of pulse time (C0) was the best pulse time for minimising surface roughness. From the $\mathrm{A} \times \mathrm{B}$ two-way table (see Appendix), the optimum combination of factors $\mathrm{A}$ and $\mathrm{B}$ in order to achieve the lowest surface roughness value was determined as A2B0. Therefore, the best combination of input variables for minimising surface roughness was specified to be A2B0C0. The variation in the surface roughness for three input parameters is shown in Figure 7. The good results are obtained when three reinforcement sizes are $13 \mu \mathrm{m}$ to gives best result), 3 and $0.7 \mu \mathrm{m}$. High and low wire tensions produce similar and superior surface finish compare to that of medium wire tension. Low pulse on time gives the best surface finish, which is then followed by high and medium pulse on times. These results are very comparable to those obtained from Pareto ANOVA and response tables.

Table 8 Pareto ANOVA for surface roughness

\begin{tabular}{|c|c|c|c|c|c|c|c|c|c|}
\hline \multirow{2}{*}{$\begin{array}{l}\text { Sum at factor } \\
\text { level }\end{array}$} & \multicolumn{9}{|c|}{ Factor and interaction } \\
\hline & A & $\mathrm{B}$ & $\mathrm{A} \times \mathrm{B}$ & $A \times B$ & $\mathrm{C}$ & $\mathrm{A} \times \mathrm{C}$ & $\mathrm{A} \times \mathrm{C}$ & $\mathrm{B} \times \mathrm{C}$ & $\mathrm{B} \times \mathrm{C}$ \\
\hline 0 & -71.42 & -64.62 & -64.95 & -68.71 & -59.72 & -66.02 & -65.90 & -65.06 & -65.81 \\
\hline 1 & -67.78 & -69.73 & -65.48 & -63.37 & -70.28 & -65.16 & -67.42 & -64.64 & -66.48 \\
\hline 2 & -60.04 & -64.89 & -68.81 & -67.16 & -69.23 & -68.05 & -65.92 & -69.54 & -66.95 \\
\hline $\begin{array}{l}\text { Sum of squares of } \\
\text { difference }(S)\end{array}$ & 202.55 & 49.64 & 26.30 & 45.23 & 203.35 & 13.18 & 4.54 & 44.35 & 1.98 \\
\hline $\begin{array}{l}\text { Contribution ratio } \\
(\%)\end{array}$ & 34.27 & 8.40 & 4.45 & 7.65 & 34.40 & 2.23 & 0.77 & 7.50 & 0.34 \\
\hline
\end{tabular}


Cumulative contribution

Check on significant interaction

Optimum combination of significant factor level

Table 9 Response table for mean $\mathrm{S} / \mathrm{N}$ ratios for surface roughness and significant interactions

\begin{tabular}{llllll}
\hline Input parameter & Symbol & Level 0 & Level 1 & Level 2 & max-min \\
\hline Particle size & $\mathrm{A}$ & -7.94 & -7.53 & -6.67 & 1.26 \\
Wire Tension & $\mathrm{B}$ & -7.18 & -7.75 & -7.21 & 0.57 \\
Pulse on Time & $\mathrm{C}$ & -6.64 & -7.81 & -7.69 & 1.17 \\
Interaction & $\mathrm{AXB}$ & -7.63 & -7.04 & -7.46 & 0.59 \\
\hline
\end{tabular}

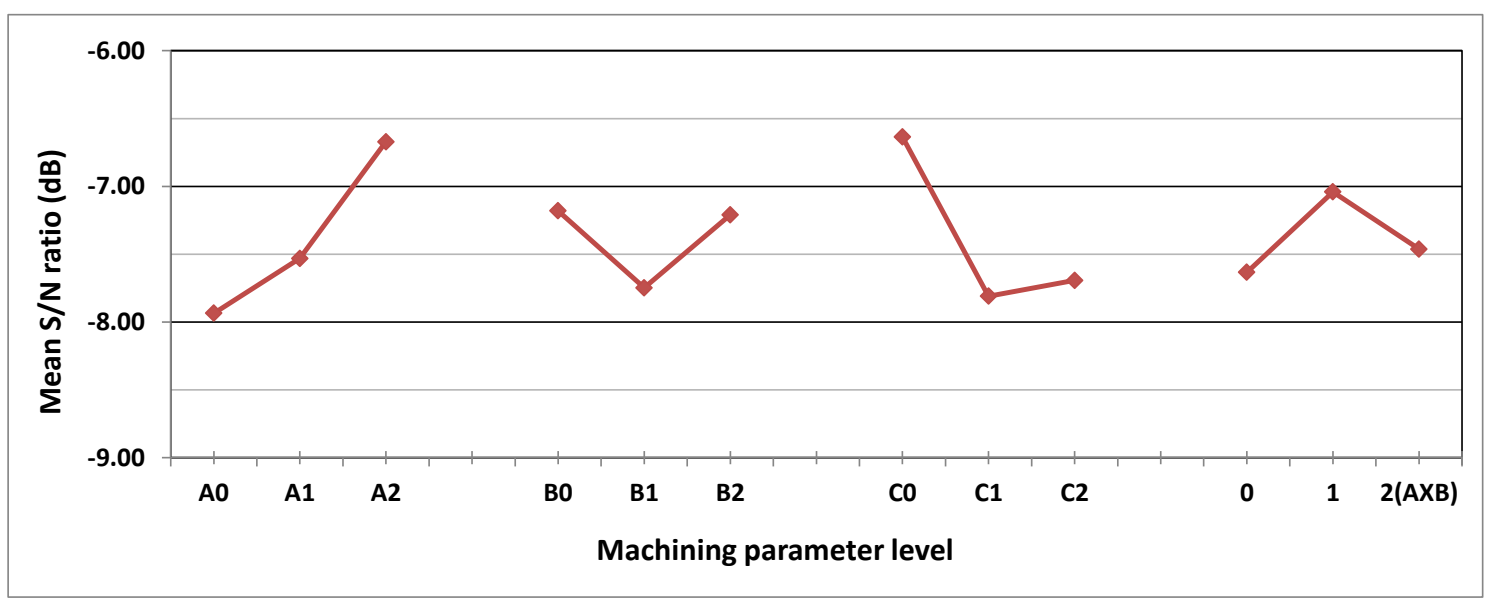

Fig. 6 Response graph of S/N ratios for Surface Roughness 


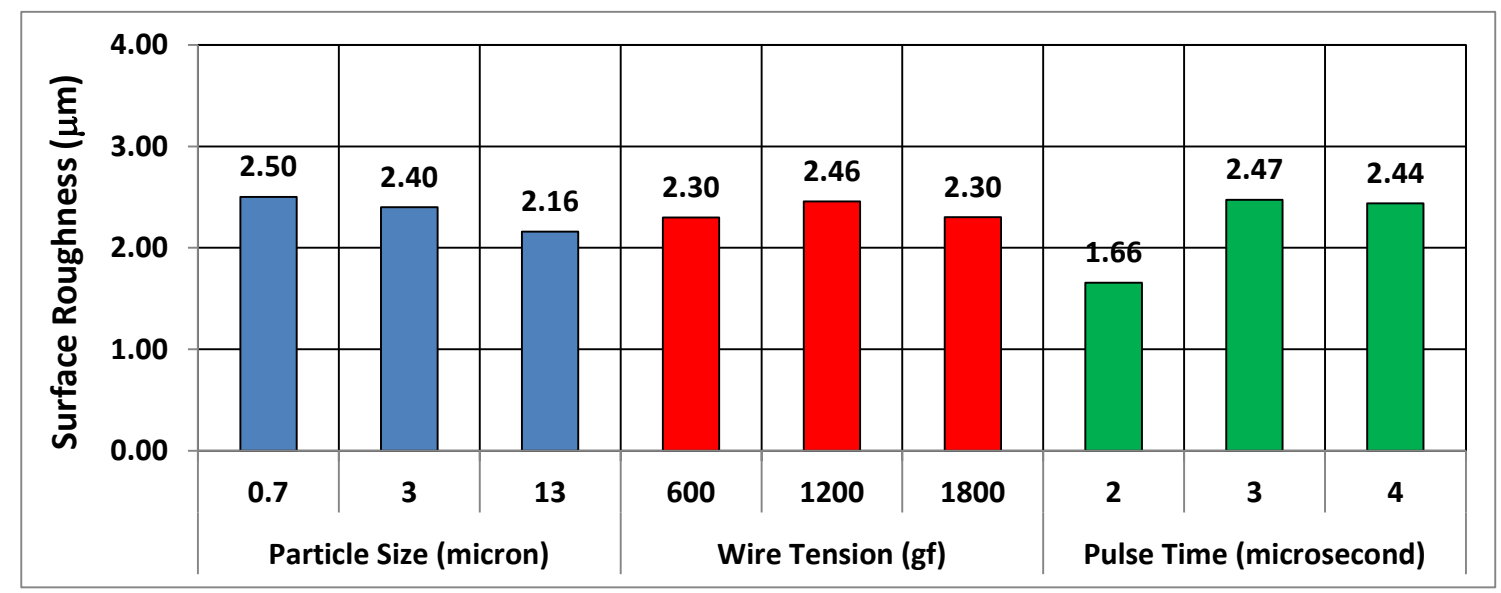

Fig. 7 Average variation of surface roughness for three additional parameters

\section{Discussion}

The results presented above indicate that input factors including size of reinforced particles, wire tension, and pulse on time showed significant effects on diameter error, circularity and surface roughness (Figures 3, 5 and 7). For the three WEDM input parameters considered, pulse on time interaction (C), wire tension (B) and pulse on time (C) are the major contributors to diameter error, circularity and surface roughness (Tables 4, 6 and 8), respectively. Reports of (i) overcut due to high temperature and electrode gap (ii) taper kerf due to deformation of wire (iii) surface damage due to formation of cavities by splashes of molten material have been published [20-24], as shown in Fig 8. Fig. 8 demonstrates that all surfaces consist of solidified melted materials, with craters due to sparks and blisters resulting from the splashing of the molten metals. There are also reinforced particles on machined surfaces of MMCs, as evidence by EDAX spectra. The presence of $\mathrm{Si}$ in the machined surface indicates that it diffuses from SiC particles at high temperatures. On the other hand, the presence of $\mathrm{Zn}$ and $\mathrm{Cu}$ indicates the transfer of wire electrode (WE) material to the workpiece by spattering. The weight fraction of transferred elements depends on $\mathrm{SiC}$ particle size in MMCs. For example, the amount of $\mathrm{Si}$ is highest when the particle size is largest. However, when the size of SiC particles was smaller (3 and $0.7 \mu \mathrm{m})$, the amount of Si on the machined surface was slightly higher for smaller particles compare to bigger particles. The reason for this is unknown and further research is required to explain this. The presence of $\mathrm{Cu}$ is also noted in this case. When the particle size decreases, the weight fraction of Si decreases as well, and Cu disappears, but the amount of $\mathrm{Zn}$ increases. All these are affected by input parameters that influence diameter error, circularity and surface roughness [23]. 

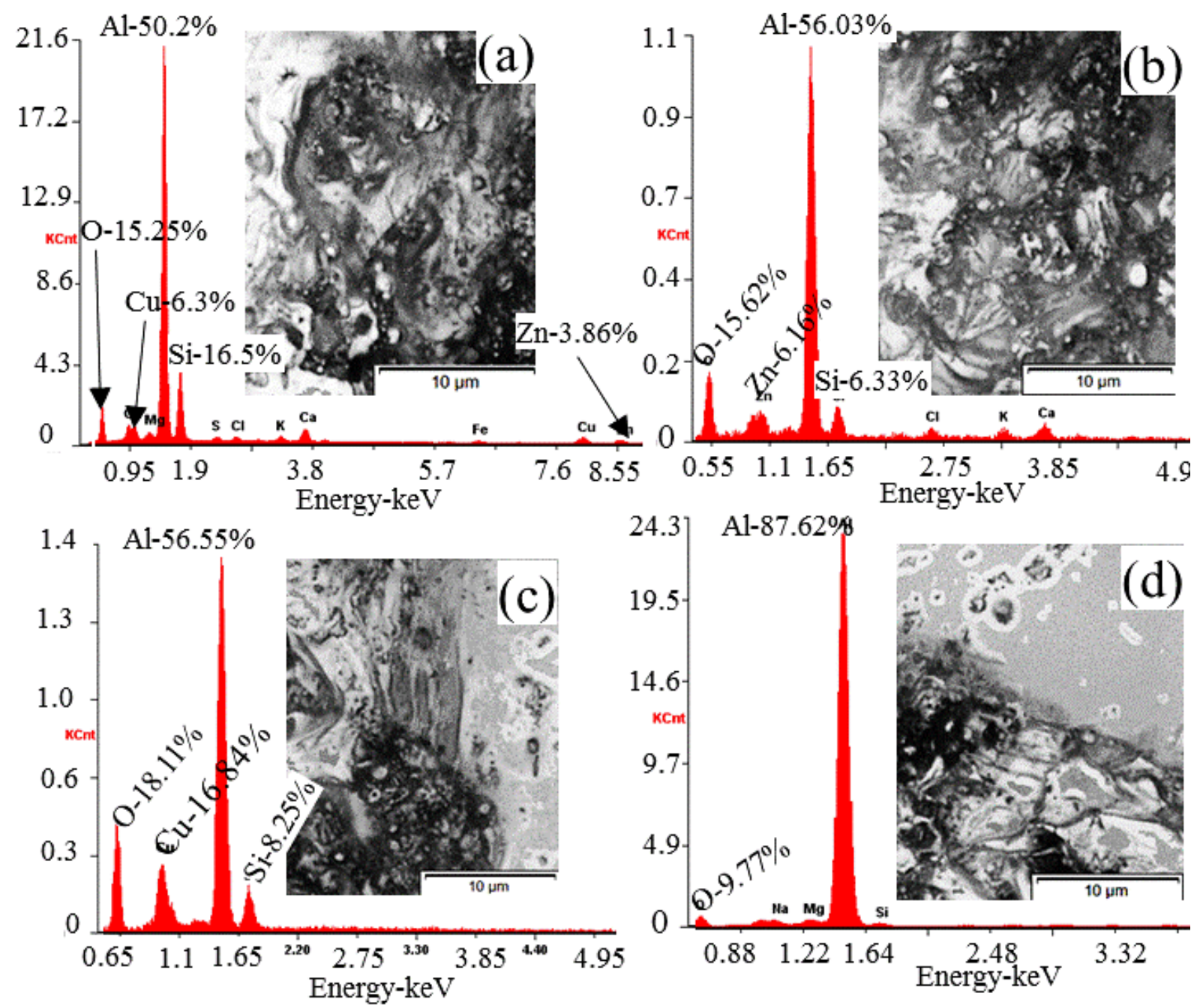

Fig. 8 Surfaces of particle sizes including (a) 13 , (b) 3 , and (c) $0.7 \mu \mathrm{m}$ and (d) matrix material after EDM [23].

The diameter error can be affected in numerous ways, such as by altering elastic deformation of the workpiece, induced by thermal distortion, projections of reinforced particles, wire electrode deformation and flexibility of wire electrode. The thermal distortion of the workpiece largely depends on (i) pulse on time and (ii) composition of MMCs and mismatch of thermal properties between matrix and reinforcement material. However, the wire tension affects the accuracy in following the cutting path and the interaction between reinforced particles and wire electrode. Therefore, the effect of interaction between reinforcement size and pulse on time $(\mathrm{A} \times \mathrm{C})$ also contributes significantly to diameter error as shown in Table 4. Though the bigger reinforcements in MMCs have higher capacity to resist the temperature, it is generally known that smaller particles can provide less disturbance of the wire movement from the cutting path, thus with less diameter error (Fig. 2). The flexibility and rigidity of the wire electrode depends on the tension in the wire, which is the second contributing factor to the diameter 
error. As the active cutting length of the wire electrode increases, the tension in the wire is reduced, resulting in a smoother cut. It is expected when the tension in the wire is less, especially in presence of MMCs reinforced with smaller particles. Consequently, diameter error is smaller with a low wire tension. As previously mentioned, the pulse on time is directly related to heat generated during EDM where longer pulse on time generates higher temperature. This temperature facilitates the vaporization of workpiece materials and wire electrode. When the pulse on time is shorter and the temperature becomes lower, the rate of vaporization is lower with less wire deformation. This observation reveals that the wire cuts less material while keeping the required wire electrode gap. Thus, the diameter error becomes the minimum at low wire tension with an optimized thermal compatibility between the phases of MMCs.

Overcut and undercut are the primary causes of circularity error, which are affected by wire tension as well as pulse on time. Therefore, the wire tension is most significant contributing factor to circularity error. The interaction between wire tension and pulse on time is the second most significant contributing factor to circularity (Table 6). At low wire tension, too flexible wire makes a longer active cutting length, which can deviate from the actual path of machining. At the high wire tension, the wire becomes very rigid and interacts with reinforced particles and pulls embedded particles in softer matrices, resulting in the poor circularity of machined holes. Therefore, the best configuration is to remove material close to the shape of a circle at the medium wire tension. The pulse on time controls the heat generation. Workpiece material does not move properly at low pulse on time due to less heat. On the other hand, too much material is removed at high pulse on time due to high heat. Therefore, medium pulse on time gives the best circularity. Where reinforced particles are small, they do not exhibit much capacity to withstand the EDM temperature, as the particles dislodge from the matrices with a low melting point, thus producing an improved circular hole. The capability to tolerate the EDM temperature improves with the increase in particle size. Further increases of particle size offer significant resistance to partial material removal. It was found that the circularity decreases when the particle size was high. Therefore, particles with a medium size balance between undercut and overcut leading to the highest circularity.

The EDM machined surfaces are generally full of solidified melted metals, splatter and blisters [22, 24]. Consequently, surface roughness has to be controlled by input energy and workpiece material. The pulse on time is the main provider to heat input, which is considered as the highest contributor to surface roughness, as depicted in table 8 . The variation of surface geometry is controlled by the particle size. The higher pulse on time leads to higher heat and more time for the surface degradation. Therefore, low pulse 
on time gives better surface finish, and the contribution of wire tension on surface roughness appears to be insignificant (Table 8). The interaction between reinforcement size and pulse on time contributes significantly to the surface finish. This clearly shows that the large reinforcement size gives the lowest surface roughness. As previously stated, when the particles are smaller, the resistance of MMCs to high temperature is weaker. More surface defects generated when particle sizes are smaller. With the increase in particle size, the surface defects are reduced in that the bigger particles increase the resistance melting of the surface. Accordingly, particles with the larger size lead to best surface finish, as exhibited in Figs. 6 and 7. It is also shown that low wire tension gives better surface finish. It is has already been mentioned that the wire is too flexible and makes a longer active cutting length at the low wire tension. This may deviate the wire from the actual path of machining, but give a smoother finish. On the other hand, the wire becomes very rigid and interacts with reinforced particles and pulls particles that are embedded in the softer matrices at the high wire tension. This results in the poor circularity and surface finish for the machined hole.

\section{Conclusion}

The above-mentioned analysis demonstrates that the reinforced particle size, wire tension, and pulse on time significantly affect diameter error, circularity and surface roughness. The following conclusions can be drawn from this investigation:

- Pulse on time and the interaction between pulse on time and wire tension contributes most significantly to diameter error. The flexibility and rigidity of the wire electrode depends on the tension. Thus, the wire tension affects the accuracy of cutting path and the interaction between reinforced particles and wire electrode. A smoother cut is expected when the wire tension is less. Though the bigger reinforcements in MMCs have the higher capacity to resist temperature, it is evident that smaller particles offer less disturbance of the wire movement from the cutting path, thus with less diameter error. The diameter error is the least at the low wire tension with an optimized thermal compatibility between the phases of MMCs.

- The wire tension is the foremost contributing factor to circularity. The interaction between wire tension and pulse on time is the second contributing factor in this case. The combination of over and undercuts are the main reason of circularity error. Best circularity was obtained at the medium particle size, medium wire tension and medium pulse on time. 
- The high size of reinforcement gives the lowest surface roughness. It has been found that more surface defects are generated when the particle sizes are smaller. With the increase of particle size, the surface defects diminish as the bigger particles increase the resistance melting of the surface. The higher pulse on time leads to higher heat and more time to degrade the surface. Therefore, low pulse on time gives better surface finish, and the contribution of wire tension on surface roughness appears to be insignificant.

\section{References}

1. Pramanik, A., Electrical discharge machining of MMCs reinforced with very small particles. Materials and Manufacturing Processes, 2016. 31(4): p. 397-404.

2. Liao, Y., J. Huang, and Y. Chen, A study to achieve a fine surface finish in Wire-EDM. Journal of Materials Processing Technology, 2004. 149(1): p. 165-171.

3. Pramanik, A. and A. Basak, Degradation of wire electrode during electrical discharge machining of metal matrix composites. Wear, 2016. 346: p. 124-131.

4. Boswell, B., et al., Effect of machining parameters on the surface finish of a metal matrix composite under dry cutting conditions. Proceedings of the Institution of Mechanical Engineers, Part B: Journal of Engineering Manufacture, 2015: p. 0954405415583776.

5. Pramanik, A., J. Arsecularatne, and L. Zhang, Machining of particulate-reinforced metal matrix composites, in Machining. 2008, Springer. p. 127-166.

6. Garg, R., et al., Review of research work in sinking EDM and WEDM on metal matrix composite materials. The International Journal of Advanced Manufacturing Technology, 2010. 50(5-8): p. 611-624.

7. Sivam, S.P., et al., Effects of electrical parameters, its interaction and tool geometry in EDM of titanium grade 5 alloy with graphite tool. Proceedings of the Institution of Mechanical Engineers, Part B: Journal of Engineering Manufacture, 2012: p. 0954405412466213.

8. Giridharan, A. and G. Samuel, Analysis on the effect of discharge energy on machining characteristics of wire electrical discharge turning process. Proceedings of the Institution of Mechanical Engineers, Part B: Journal of Engineering Manufacture, 2015: p. 0954405415615732.

9. Mouangue N, A., et al., Effects of Electro-discharge Machining on Aluminium Metal Matrix Composite. Journal of Applied Sciences, 2011. 11: p. 1668-1672.

10. Singh, P.N., et al., Electric discharge machining of Al-10\% SiC $p$ as-cast metal matrix composites. Journal of Materials Processing Technology, 2004. 155: p. 1653-1657.

11. Khan, E.F. and E.B. Singh, Machining of Al/Sic MMC on EDM. International Journal of IT, Engineering and Applied Sciences Research, 2012. 1(2): p. 28-30.

12. Lal, S., et al., Optimization of wire electrical discharge machining process parameters on material removal rate for Al7075/SiC/Al2O3 hybrid composite. Proceedings of the 
Institution of Mechanical Engineers, Part B: Journal of Engineering Manufacture, 2014: p. 0954405414533511.

13. Tian, X., et al., Sensitivity thermal analysis of electrical discharge machining process based on probabilistic design system. Proceedings of the Institution of Mechanical Engineers, Part B: Journal of Engineering Manufacture, 2015. 229(5): p. 813-822.

14. Amini, H., M.S. Yazdi, and G. Dehghan, Optimization of process parameters in wire electrical discharge machining of TiB2 nanocomposite ceramic. Proceedings of the Institution of Mechanical Engineers, Part B: Journal of Engineering Manufacture, 2011: p. 0954405411412249.

15. Rozenek, M., et al., Electrical discharge machining characteristics of metal matrix composites. Journal of Materials Processing Technology, 2001. 109(3): p. 367-370.

16. ASME, Dimensioning and Tolerancing, in ASME. 2009, ASME: New York.

17. Park, S., Robust design and analysis for quality engineering. 1996: Boom Koninklijke Uitgevers.

18. Ross, P.J., Taguchi techniques for quality engineering. Mcgraw-hil International editions, 1996.

19. Taguchi, G. and G. Taguchi, System of experimental design; engineering methods to optimize quality and minimize costs. 1987.

20. Tosun, N. and C. Cogun, An investigation on wire wear in WEDM. Journal of materials processing technology, 2003. 134(3): p. 273-278.

21. Pramanik, A., Developments in the non-traditional machining of particle reinforced metal matrix composites. International Journal of Machine Tools and Manufacture, 2014. 86: p. 44-61.

22. Pramanik, A., Electrical Discharge Machining of MMCs Reinforced with Very Small Particles. Materials and Manufacturing Processes, 2015(just-accepted): p. DOI: 10.1080/10426914.2015.1048360.

23. Pramanik, A., A. Basak, and M. Islam, Effect of reinforced particle size on wire EDM of MMCs. International Journal of Machining and Machinability of Materials, 2015. 17(2): p. 139-149.

24. Pramanik, A., et al., Electrical discharge machining of 6061 aluminium alloy. Transactions of Nonferrous Metals Society of China, 2015. 25(9): p. 2866-2874. 


\section{Appendix}

$\mathrm{BC}$ two-way table for diamtere error

\begin{tabular}{|c|c|c|c|c|c|c|c|c|c|c|c|c|c|}
\hline & & BO & & & & B1 & & & & B2 & & & Total \\
\hline CO & 22.536 & 21.441 & 23.060 & $\underline{67.037}$ & 21.793 & 20.383 & 21.373 & 63.549 & 21.582 & 20.412 & 20.934 & 62.928 & 130.586 \\
\hline C1 & 21.343 & 20.565 & 20.439 & 62.348 & 20.946 & 19.856 & 20.915 & 61.716 & 21.010 & 20.115 & 20.413 & 61.538 & 185.602 \\
\hline C2 & 20.754 & 18.180 & 19.910 & 58.844 & 20.354 & 23.963 & 20.506 & 64.823 & 20.598 & 19.574 & 20.028 & 60.200 & 183.867 \\
\hline Total & & & & 188.229 & & & & 190.088 & & & & 184.666 & 500.055 \\
\hline
\end{tabular}

BOCO has the highest value, therefore it is the best combination

BC two-way table for circularity

\begin{tabular}{|c|c|c|c|c|c|c|c|c|c|c|c|c|c|}
\hline & & BO & & & & B1 & & & & B2 & & & Total \\
\hline CO & 44.815 & 44.815 & 44.357 & 133.987 & 41.192 & 42.007 & 38.633 & 121.832 & 43.943 & 41.893 & 37.764 & 123.600 & 379.419 \\
\hline C1 & 41.761 & 44.397 & 41.192 & 127.350 & 43.039 & 23.597 & 35.912 & 102.548 & 43.098 & 42.570 & 43.010 & 128.678 & 358.576 \\
\hline C2 & 42.194 & 39.148 & 41.407 & 122.749 & 43.436 & 38.396 & 33.556 & 115.388 & 41.871 & 42.243 & 43.500 & 127.614 & 365.751 \\
\hline Total & & & & 384.086 & & & & 339.768 & & & & 379.892 & 1103.746 \\
\hline
\end{tabular}

BOCO has the highest value, therefore it is the best combination

$A B$ two-way table foe surface roughness

\begin{tabular}{|c|c|c|c|c|c|c|c|c|c|c|c|c|c|}
\hline & & AO & & & & A1 & & & & A2 & & & Total \\
\hline BO & -6.550 & -8.538 & -8.397 & -23.484 & -6.426 & -6.945 & -8.563 & -21.934 & -5.678 & -6.779 & -6.743 & $\underline{-19.201}$ & -64.619 \\
\hline B1 & -7.217 & -7.674 & -8.695 & -23.587 & -6.691 & -9.822 & -8.749 & -25.262 & -8.563 & -7.880 & -6.309 & -22.753 & -71.602 \\
\hline B2 & -7.507 & -8.803 & -8.036 & -24.347 & -6.663 & -7.493 & -6.426 & -20.582 & -6.293 & -6.351 & -7.315 & -19.959 & -64.888 \\
\hline Total & & & & -71.417 & & & & -67.779 & & & & -61.912 & -201.108 \\
\hline
\end{tabular}

$\mathrm{A} 2 \mathrm{BO}$ has the highest value, therefore it is the best combination 